\title{
Further evidence on the abstraction of linguistic ideas
}

\author{
JOHN T. E. RICHARDSON \\ Brunel University, Uxbridge, Middlesex, England
}

\begin{abstract}
Three accounts of the processes underlying the retention of connected sentences expressing complex linguistic ideas were empirically compared: an integrationist model, an imagery model, and a tally model. The reaction time in a test of recognition memory was found to be independent of the number of components expressed by a sentence, which was inconsistent with the tally model. Concrete ideas and abstract ideas both showed complete integration, which was inconsistent with the imagery model. The integrationist approach was regarded as the most useful model of the abstraction of complex linguistic ideas.
\end{abstract}

Bransford and Franks (1971) investigated the retention of sets of sentences that together defined complex linguistic ideas. For instance, the complex idea "The ants in the kitchen ate the sweet jelly which was on the table" consisted of the elements "The ants were in the kitchen," "The jelly was on the table," "The jelly was sweet," and "The ants ate the jelly." The acquisition sentences in the original experiment contained one, two, or three elements from any one of four distinct ideas. After an intervening task, the subjects received a recognition test with sentences containing one, two, three, or four elements of each idea. The subjects' confidence that a particular sentence had been presented for learning varied directly with the number of elements that it contained. A second experiment showed that such confidence ratings were largely independent of whether a sentence had actually been presented, which Bransford and Franks took to mean that the subjects had abstracted the information contained in sets of individual acquisition sentences to construct holistic semantic ideas. Moreover, the correlation between the confidence rating assigned to a test sentence and the number of elements that it contained suggested that recognition judgments might be based upon the degree of overlap between a test sentence and a complete idea.

These findings were subsequently replicated by Bransford and Franks (1972) and by others (Cofer, 1973; Griggs, 1974; Katz, 1973; Katz, Atkeson, \& Lee, 1974; Singer, 1973). However, the sentences used in these studies typically described concrete events or situations and readily evoked mental imagery. Consequently, as Neisser (1972) observed, it is interesting to

The author is grateful for the assistance of Sean Chamberlain, Ignatius Chew, John Doherty, Stuart Gibson, Jeffrey Hooper, and Colin Rafferty in running this experiment. Requests for reprints should be addressed to John T. E. Richardson, Department of Psychology, Brunel University, Uxbridge, Middlesex UB8 3PH, England. consider whether a holistic semantic idea might consist, in whole or in part, in a composite mental image. Begg and Paivio (1969) argued that concrete sentences were stored as mental images, whereas abstract sentences were stored in a relatively superficial form, "as implicit auditory-motor representations" (Paivio, 1971a, 1971b, p. 53). It follows that the abstraction of information in the form of holistic ideas should be difficult or impossible in the case of abstract material. Using abstract ideas Franks and Bransford (1972) repeated their original experiments and obtained essentially the same results. This indicated that the synthesis of information occurred with both concrete and abstract material, and did not depend upon the concretization of that information in the form of mental imagery (cf. Bransford, Barclay, \& Franks, 1972).

However, the question of whether concreteness affects the integrative process can really only be resolved by comparing concrete and abstract material within the same experiment. Moeser (1975) reported one such investigation, and concluded that concrete sentences were more easily integrated into holistic ideas than were abstract sentences. However, there are several problems with Moeser's study. First, none of the acquisition sentences was presented during the recognition phase; all of the test sentences were actually "new." As Moeser noted, this forced the subjects to adopt an "acquiescent response bias" that might have distorted the experimental outcome. Second, her subjects had to say whether the test sentences were "identical" or "changed," not whether they were "old" or "new." Since the instructions did not distinguish between verbatim identity and synonymy, the judgment to be made was left unclear. Third, Moeser presented no evidence to show that she had effectively manipulated stimulus concreteness in her choice of material, or that she had properly controlled the intelligibility of the sentences to be learned. The study to be reported in the present paper attempted to remedy these deficiencies. 
Reitman and Bower (1973) argued that Bransford and Franks's (1971) results could be handled by a tally theory, according to which a complex idea was represented in memory by all of its possible components, with each such component being tagged according to its occurrence in the acquisition phase. Thus, if a particular acquisition item containing three elements were represented by the expression $A B C$, the individual elements ( $\mathrm{A}, \mathrm{B}$, and $\mathrm{C})$, the possible digrams ( $\mathrm{AB}, \mathrm{AC}$, and $\mathrm{BC})$, and the trigram itself $(\mathrm{ABC})$ would be tagged. In the recognition test, each element of a test sentence would be inspected, and the item rejected as "new" if the number of tags on any one element were below a certain threshold. Otherwise, and if the test sentence contained two or more elements, each possible digram would be inspected. If the number of tags on a given digram exceeded a threshold, a tally would be added to a counter for that test sentence; if not, a tally would be subtracted. Similarly, if the test sentence contained three or more elements, each possible trigram would be inspected. The subject's final confidence rating was assumed to be a linear transformation of the accumulated tally for the test sentence. Reitman and Bower assumed that every element in a test sentence would be recognized, and they set the probability of the number of tags on a given n-gram exceeding the tally threshold at a constant, $p$. The expected total tally for a sentence that contains $\mathrm{m}$ elements of a complex idea may be shown to be equal to $(2 p-1)(2 m-m-1)$. (This expression differs from the formula given by Reitman and Bower, which is arbitrarily and inexplicably restricted to n-grams of adjacent elements.) For the original experiments of Bransford and Franks, the values of $p$ for old and new test sentences might be expected to be similar and greater than .5. In this case, the functions relating confidence ratings to the number of elements in a test sentence would also be similar and would have positive slopes, as indeed was found by Bransford and Franks. Thus, a radically different sort of model is capable of handling the empirical effects that were originally attributed to the processes of semantic integration.

Reitman and Bower (1973) were able to demonstrate similar effects using nonsemantic alphanumeric character strings as their stimulus vocabulary; this suggested that the processes responsible for those effects were not essentially semantic in nature. Similarly, Katz et al. (1974) referred to an unpublished study by Katz and Gruenewald that produced the same basic results with semantically anomalous sentences. Katz et al. showed further that test sentences containing one element of a complex idea would produce substantially higher confidence ratings if the acquisition sentences had themselves only contained one element. Subsequently, Flagg (1976) and Pezdek (1978) showed that a negative slope in the confidence-rating function would occur if the complex ideas were presented during the acquisition phase as sentences containing only one of the relevant elements. These results were taken to provide additional support for the tally theory and to be inconsistent with the integrationist model proposed by Bransford and Franks. In truth, the latter authors had actually predicted such findings on the grounds that the longer test sentences would in such cases be rejected as inconsistent with the general style of the acquisition material. It is, in any event, quite unsurprising that the processes that determine recognition judgments should be affected by variations in the experimental procedure. For instance, it is apparent that the confidence-rating function has zero slope when subjects are asked to judge whether the test sentences have the same meaning as the acquisition sentences (Katz, 1973; Moeser, 1975).

Fortunately, Reitman and Bower (1973) themselves pointed out a much less equivocal test of their theory. A difficulty with the tally model is that it involves a considerable amount of computation before a recognition judgment can be made; in particular, "the reaction time for production or recognition of an item which fit the full concept would be unreasonably longer than for any presented subunit." In fact, it is easy to show that the number of n-grams to be interrogated for a test sentence containing $\mathrm{m}$ elements of a complex idea is equal to $\left(2^{\mathrm{m}}-1\right)$. Thus, the tally theory leads to the clear but counterintuitive prediction that the latency of a recognition judgment in Bransford and Franks's paradigm should be a direct and positively accelerating function of the number of elements in a test sentence. On the other hand, neither the integrationist theory of Bransford and Franks nor the imageability theory of Begg and Paivio (1969) predicts such a relationship.

\section{METHOD}

\section{Subjects}

Twenty-four undergraduates from different courses at Brunel University volunteered to participate in this experiment.

\section{Materials}

Four concrete ideas were taken from those used by Bransford and Franks (1971), and four abstract ideas were taken from those used by Franks and Bransford (1972). Separate groups, each of five subjects, had previously rated these sentences along 7-point scales in terms of their imageability and their intelligibility, using instructions adapted from those of Paivio, Yuille, and Madigan (1968). The mean rated imageability of the concrete and abstract sentences was 6.20 and 3.45 , respectively, and their mean rated intelligibility was 5.40 and 5.40 , respectively.

With each complex idea being represented by the expression $A B C D$, there are 12 possible sentences expressing one or more of the four elements: $\mathrm{A}, \mathrm{B}, \mathrm{C}, \mathrm{D}, \mathrm{AB}, \mathrm{AC}, \mathrm{BC}, \mathrm{CD}, \mathrm{ABC}, \mathrm{ACD}$, $B C D$, and $A B C D$. One acquisition list was formed by taking sentences $\mathrm{A}, \mathrm{C}, \mathrm{BC}, \mathrm{CD}$, and $\mathrm{ABC}$ from two concrete and two abstract ideas, and sentences $\mathrm{B}, \mathrm{D}, \mathrm{AB}, \mathrm{AC}$, and $\mathrm{BCD}$ from the remainder. A second acquisition list was formed in the reverse fashion. The recognition list consisted of the two acquisition lists combined.

\section{Procedure}

The subjects were tested individually. They were instructed to try to remember $\mathbf{4 0}$ sentences in an acquisition list, but they were given no specific mnemonic instructions and were not 
informed of the structure of the list. The sentences were presented visually, typed on plain white index cards, at a rate of 1 every $10 \mathrm{sec}$. Half of the subjects received each acquisition list, and the sentences in each list were presented in a different random order to each subject. Each subject was then instructed to carry out an irrelevant task for $5 \mathrm{~min}$; the task required that the subject cancel any item that was divisible by 3 or 4 from a random array of two-digit numbers. Half of the subjects who had received each acquisition list were then presented with the 80 sentences in the recognition list and were asked to judge whether each had been shown earlier and to indicate their confidence in that judgment on a numbered 5-point scale. The sentences, again typed on plain white index cards, were presented in a different random order to each subject. The responses were written on prepared response sheets, and the subjects worked through the recognition list without time pressure. The remaining subjects were presented with the 80 recognition sentences through a two-field tachistoscope and were asked to press one of two response keys to indicate whether each had been shown earlier. Half of these subjects made an affirmative response with their left hands and a negative response with their right hands, and the pattern was reversed for the remainder. The sentences were presented in a different random order to each subject, who was instructed to "press one of the buttons as quickly as possible, but make sure that it is the response you want to make before you do so." An electronic timer measured the latency of the subjects' responses in milliseconds.

\section{RESULTS}

All 24 subjects produced data on the frequency of "yes" and "no" responses. Table 1 shows the mean percentage of affirmative responses for old and new, concrete and abstract sentences expressing one, two, or three elements of a complex idea. The likelihood of a "yes" response was directly related to the number of elements; there was no discrimination between old and new test sentences, except for those containing one element; and the concreteness of the material had no effect at all upon the pattern of performance. These effects were examined by means of an analysis of vari-

Table 1

Mean Percentage Affirmative Responses, Mean Confidence Ratings, and Harmonic Mean Response Latencies (in Seconds)

\begin{tabular}{|c|c|c|c|c|}
\hline & & \multicolumn{3}{|c|}{ Number of Elements } \\
\hline & & 1 & 2 & 3 \\
\hline \multicolumn{5}{|c|}{ Mean Percentage Affirmative Responses } \\
\hline \multirow{2}{*}{ Concrete } & Old & 54.0 & 61.4 & 63.6 \\
\hline & New & 40.3 & 56.8 & 62.5 \\
\hline \multirow{2}{*}{ Abstract } & Old & 55.1 & 60.8 & 64.8 \\
\hline & New & 38.1 & 55.1 & 63.6 \\
\hline \multicolumn{5}{|c|}{ Mean Confidence Ratings } \\
\hline \multirow[b]{2}{*}{ Concrete } & Old & +.59 & +.55 & +.10 \\
\hline & New & -.59 & +.29 & +.15 \\
\hline \multirow{2}{*}{ Abstract } & Old & +.53 & +.70 & +1.05 \\
\hline & New & -.43 & +1.13 & +1.35 \\
\hline \multicolumn{5}{|c|}{ Harmonic Mean Response Latencies } \\
\hline \multirow{2}{*}{ Concrete } & Old & 1.54 & 1.73 & 1.83 \\
\hline & New & 1.63 & 1.81 & 2.00 \\
\hline \multirow{2}{*}{ Abstract } & Old & 1.56 & 1.76 & 2.04 \\
\hline & New & 1.55 & 1.91 & 2.11 \\
\hline
\end{tabular}

ance, whose basic design contained two random factors, subjects and ideas, and two fixed factors, old versus new sentences and the number of elements. The first random factor was nested under the fixed factors of groups (rating subjects vs. latency subjects) and acquisition lists, and the second random factor was nested under the fixed factor of concreteness. The effect of the number of elements in a test sentence was partitioned into linear and quadratic contrasts. The only significant $F$ ratio was that associated with the linear effect of the number of elements [quasi $F(1,12)=7.04, p<.025$ ], although its interaction with the comparison between old and new sentences approached significance $[F(1,20)=3.20$, $\mathrm{p}<.1$ ]. The latter main effect was not significant [quasi $\mathrm{F}(1,14)=2.52, \mathrm{p}>.1$ ], and a posteriori tests showed that the comparison was significant for sentences containing one element $[F(1,18)=6.80, p<.02]$, but not for sentences containing two elements $[F(1,18)$ $=1.02, \mathrm{p}>3]$ or for sentences containing three elements $(F<1)$. Conversely, the linear effect of the number of elements was significant for new sentences $[F(1,12)=11.81, p<.005]$, but not for old sentences $[\mathrm{F}(1,12)=2.68, \mathrm{p}>.1]$.

Half of the subjects produced confidence ratings of their recognition judgments; following established practice, these were encoded as numerical values by assigning a "plus" to an affirmative response and a "minus" to a negative response. Table 1 shows the mean recognition ratings for old and new, concrete and abstract sentences expressing one, two, or three elements of a complex idea. The ratings were directly related to the number of elements in a test sentence; there was little discrimination between old and new sentences, except for those containing one element; and there was no clear effect of stimulus concreteness. Unfortunately, an analysis of variance failed to show any significant main effects or interactions among the fixed factors of interest, although the linear effect of the number of elements approached statistical significance on a directional test [quasi $F(1,16)=1.82, p<.1$ ] . It is possible that the large number of response categories increased the amount of error variation in these data.

The remaining 12 subjects produced reaction time measures of their recognition judgments. These data clearly followed a skewed distribution, and therefore reciprocals of the original observations were used for statistical analysis. Accordingly, Table 1 shows the harmonic mean response latencies for old and new, concrete and abstract sentences expressing one, two, or three elements of a complex idea. Response latencies increased with the number of elements in a test sentence, but there was little difference between old and new sentences or between concrete and abstract sentences. An analysis of variance was carried out upon the transformed data, using the same design as for the analysis of affirmative responses, except that the random factor of subjects was nested under the fixed factors of response pattern (left hand vs. right hand for affirmative 
responses) and acquisition lists. The only significant $\mathrm{F}$ ratio was that associated with the linear effect of the number of elements [quasi $\mathrm{F}(1,13)=10.57, \mathrm{p}<.01$ ] The quadratic effect, although not significant $[F(1,8)=$ $1.60, \mathrm{p}>.2]$, was positive, reflecting the negatively accelerating function between the harmonic means and the number of elements.

Nevertheless, it is obvious that recognition sentences with more elements might produce longer latencies simply because they contain more words and thus take longer to encode. An analysis of covariance was carried out on the average reciprocal reaction times obtained with old and new sentences of one, two, and three elements expressing each of the eight ideas. The covariate was the average number of words in the corresponding sentences, and the effect of the number of elements was again partitioned into linear and quadratic contrasts. The covariate explained a significant amount of the within-idea error variation $[\mathrm{r}=-.52 ; \mathrm{F}(1,29)=$ $10.98, \mathrm{p}<.005]$, but no term in the analysis was significant when its effect was statistically controlled. In particular, the linear effect of the number of elements was no longer significant $[F(1,29)=1.34, p>.25]$, and the corrected harmonic mean response latency was actually longer for sentences expressing one element $(1.87 \mathrm{sec})$ than for sentences expressing three elements $(1.63 \mathrm{sec})$.

\section{DISCUSSION}

Reitman and Bower's (1973) tally theory of recognition memory gave rise to the clear prediction that recognition latencies in the paradigm devised by Bransford and Franks (1971) should be a direct and positively accelerating function of the number of elements in a particular test sentence. This experiment did indeed obtain such a direct relationship, but it was negatively accelerating and was entirely attributable to the fact that sentences that expressed more elements also tended to contain more words. When the length of a sentence was statistically controlled, sentences that expressed three elements of a complex idea did not produce longer latencies than those that expressed only one element. This finding is inconsistent with the particular tally theory described by Reitman and Bower, but it is also inconsistent with any theory that characterizes recognition judgments of complex stimuli in terms of computations based upon a frequency distribution of tags across all possible subunits or components of those stimuli. On the other hand, it is consistent both with the integrationist theory of Bransford and Franks (1971) and with the imagery theory of Begg and Paivio (1969).

In none of the sets of empirical data was there any sign of an effect of concreteness upon recognition performance. At least in ihe incidence of affirmative responses, both concrete and abstract material reproduced the original phenomena of a clear effect of the number of elements that were expressed in a particular sentence upon the recognition of that sentence, and a poor discrimination of old and new test sentences. The same pattern appeared in the subjects' confidence ratings, although the data exhibited much more variability. These results are inconsistent with the imagery theory proposed by Begg and Paivio (1969), which attributed the retention of concrete material to the construction of composite mental images, and the retention of abstract material to the maintenance of discrete auditorymotor representations. However, these results are entirely consistent with the integrationist theory of Bransford and Franks (1971), which assumes that the integration of semantic information does not depend upon the concretization of that information in the form of mental imagery. As Franks and Bransford (1972) concluded, irrespective of the concreteness of the stimulus material, the results appear to be best interpreted in terms of the integration of partial information and the storage of holistic representations in memory.

\section{REFERENCES}

Begg, I., \& Paivio, A. Concreteness and imagery in sentence learning. Journal of Verbal Learning and Verbal Behavior, $1969,8,821-827$.

Bransford, J. D., Barclay, J. R., \& Franks, J. J. Sentence memory: A constructive versus interpretative approach. Cognitive Psychology, 1972, 3, 193-209.

Bransford, J. D., \& Franks, J. J. the abstraction of linguistic ideas. Cognitive Psychology, 1971, 2, 331-350.

Bransford, J. D., \& Franks, J. J. The abstraction of linguistic ideas: A review. Cognition, 1972, 1, 211-249.

Cofer, C. N. Constructive processes in memory. American Scientist, 1973, 61, 537-543.

FlaGg, P. W. Semantic integration in sentence memory? Journal of Verbal Learning and Verbal Behavior, 1976, 15, 491-504.

Franks, J. J., \& Bransford, J. D. The acquisition of abstract ideas. Journal of Verbal Learning and Verbal Behavior, 1972, 11, 311-315.

Griggs, R. A. The recall of linguistic ideas. Journal of Experimental Psychology, 1974, 103, 807-809.

KATZ, S. Role of instructions in abstraction of linguistic ideas. Journal of Experimental Psychology, 1973, 98, 79-84.

Katz, S., Atkeson, B., \& LeE, J. The Bransford-Franks linear effect: Integration or artifact? Memory \& Cognition, 1974, 2, 709-713.

MoESER, S. D. The integration of verbal ideas. Canadian Journal of Psychology, 1975, 29, 106-123.

NE ISSE R, U. Changing conceptions of imagery. In P. W. Sheehan (Ed.), The function and nature of imagery. New York: Academic Press, 1972.

PAIvio, A. Imagery and language. In S. J. Segal (Ed.), Imagery: Current cognitive approaches. New York: Academic Press, 1971. (a)

PAIvio, A. Imagery and verbal processes. New York: Holt, Rinehart \& Winston, 1971. (b)

Paivio, A., Yuille, J. C., \& Madigan, S. A. Concreteness, imagery, and meaningfulness values for 925 nouns. Journal of Experimental Psychology Monograph, 1968, 76(1, Pt. 2).

Pezde K, K. Recognition memory for related pictures. Memory \& Cognition, 1978, 6, 64-69.

Reitman, J. S., \& Bower, G. H. Storage and later recognition of exemplars of concepts. Cognitive Psychology, 1973, 4, 194-206.

Singer, M. A replication of Bransford and Franks' (1971) "The abstraction of linguistic ideas." Bulletin of the Psychonomic Society, 1973, 1, 416-418.

(Manuscript received for publication August 16, 1983.) 